\title{
LES KYSTES A TETRAMERES DU PIGEON
}

\author{
Par Jean TIMON-DAVID
}

Le genre Tetrameres Diesing 1835, groupe un certain nombre d'espèces remarquables par leur dimorphisme sexuel très accusé ; tandis que le mâle se présente comme un nématode classique, la femelle ressemble à un véritable sac à œufs, irrégulièrement bosselé et sillonné. La systématique de ces vers a fait l'objet de nombreux travaux de Diesing, von Linstow, Travassos, Seurat, Crain, etc. Le cycle évolutif a été suivi dans quelques cas : le premier hôte est un crustacé (Daphnia pulex, Gammarus pulex) ; l'hôte définitif est un oiseau (albatros, cygne, canard, foulque, pigeon, flamant, héron, etc.). Les lésions déterminées par ces helminthes ont été peu étudiées et ce travail, consacré principalement à l'étude anatomo-pathologique d'un pigeon parasité, se propose de combler cette lacune.

On sait que les espèces du genre Tetrameres habitent électivement le proventricule. Les auteurs qui se sont occupés de ce nématode font remarquer que les vers mâles restent libres dans la cavité, tandis que les femelles s'enfoncent profondément dans la paroi et se logent dans les cryptes glandulaires. J'ai constaté, ainsi que je l'expose plus loin, des faits tout différents.

Je rappelle d'abord, dans cette étude, les caractères essentiels du parasite, caractères bien connus, mais dont la claire représentation est nécessaire à la compréhension de ce qui suit; je décris ensuite la structure normale du proventricule du pigeon et les lésions de la paroi parasitée.

L'oiseau qui a fait l'objet de ces recherches a été tué dans la banlieue de Marseille, à quelques kilomètres de la ville.

\section{Le parasite}

Tetrameres fissispina (Diesing 1861) Travassos 1914 doit être considéré comme synonyme dés dénominations suivantes : Tropidocerca fissispina Diesing 1861, Tropisurus fissispinus (Diesing 1861) Neumann 1888, Acanthophorus tenuis Linstow 1899, Filaria pulicis Linstow 1894, Spiroptera pulicis (Linstow 1894). 
Les deux sexes ont, comme caractères communs, une capsule buccale chitineuse, une bouche munie de trois petites lèvres et un pharynx bien musclé.

La femelle ressemble vaguement à une pyramide à quatre faces, la bouche étant située à l'extrémité apicale, la paroi du corps est plissée et dessine des sortes de circonvolutions. Sa longueur varie de 2,3 à $3 \mathrm{~mm}$. et sa largeur de 1 à $2 \mathrm{~mm}$. L'extrémité postérieure se prolonge en un court appendice caudal. Quatre sillons longitudinaux parcourent toute la largeur du ver qui est de couleur rougeâtre. La capsule buccale, plus longue que large, mesure $20 \mu$ sur $10 \mu$; elle donne accès dans un pharynx long de 220 à $240 \mu$ auquel fait suite un œsophage de $1 \mathrm{~mm}$. et un intestin globuleux ou piriforme. Les contours du tube digestif sont nettement soulignéśs sur les coupes par une masse noirâtre qui le remplit; il s'agit vraisemblablement de produits de décomposition de l'hémoglobine. Cette substance noire est parfois si abondante qu'elle est visible par transparence à travers la paroi du corps. L'anus s'ouvre à $70 \mu$ de l'extrémité caudale.

En dehors du tube digestif, les organes génitaux, ovaires, oviductes et utérus, remplissent le corps du ver. Il existe une bourse copulatrice. La vulve s'ouvre à $310 \mu$ de l'extrémité caudale. Les œufs, prodigieusement nombreux, mesurent 50 à $55 \mu$ sur 25 à $30 \mu$. Beaucoup ont déjà subi une segmentation et renferment de nombreux noyaux; ils sont pondus embryonnés.

Le mâle, long de 3 à $6 \mathrm{~mm}$., large de 140 à $150 \mu$, porte, sur les deux lignes médianes et latérales, une rangée d'épines ; les épines médianes situées entre la capsule-buccale et l'origine de l'intestin sont bifides. Il y a des papilles cervicales à $150 \mu$ de l'extrémité antérieure. La capsule buccale mesure $8 \mu$ sur $3 \mu$; l'œsophage est long de $780 \mu$; le cloaque s'ouvre à $130 \mu$ de l'extrémité postérieure; quant à la longueur du spicule, elle varie, suivant les descriptions des auteurs, de 82 à $490 \mu$. Derrière l'ouverture cloacale sont 5 épines ventrales et 3 épines latérales de chaque côté. Le corps du ver se termine par une épine de $4 \mu$.

\section{La paroi normale}

La constitution histologique du proventricule du pigeon est bien connue depuis les travaux de Leydig (1854), Hasse (1865), Nüssbaum (1877), Cattaneo (1885), Cazin (1888), etc. C'est un organe essentiellement glandulaire, pourvu d'une musculeuse peu développée et d'une muqueuse très épaisse. 
La musculeuse est composée d'une couche externe de fibres circulaires et d'une couche interne de fibres longitudinales. Postma (1877) a montré qu'il existe une troisième tunique longitudinale externe, peu développée, mais bien visible. Ces formations ont en moyenne les épaisseurs suivantes :

Couche longitudinale externe 10 à $46 \mu$.

Couche circulaire externe $150 \mu$.

Couche longitudinale interne 50 à $90 \mu$.

Quant à la muqueuse, elle atteint, avec la sous-muqueuse, une épaisseur moyenne de 2 millimètres. Elle constitue donc la partie la plus importante de l'organe.

La cavité du proventricule est tapissée d'un épithélium cylindrique simple, formé de cellules hautes de $15 \mu$. Cet épithélium se soulève en saillies et s'affaisse dans de légères dépressions, au fond desquelles viennent s'ouvrir les cryptes glandulaires.

Ce sont ces follicules glandulaires qui donnent aux coupes du proventricule leur physionomie caractéristique, de même que ce sont eux qui assument, physiologiquement le rôle le plus important. Ils se présentent comme des sacs à sommet plus ou moins conique, à contour parfois arrondi ou elliptique, mais le plus souvent polygonal. Leur hauteur est un peu variable suivant leur localisation : elle atteint $2 \mathrm{~mm}$., 5 dans la région médiane, tandis qu'elle ne dépasse $1 \mathrm{~mm} ., 3$ dans les portions qui sont voisines de l'œsophage et du gésier. Ces paquets glandulaires sont enveloppés d'une épaisse tunique conjonctive.

Chaque acinus est constitué par une cavité centrale, irrégulièrement plissée et accidentée, dans laquelle viennent déboucher les canaux collecteurs d'une multitude de glandes tubulaires. Ce vestibule commun est tapissé d'un épithélium à cellules muqueuses, qui s'étend aussi aux canaux collecteurs.

Les glandes tubulaires sont orientées radialement; elles sont composées de cellules granuleuses avec un gros noyau vésiculeux et des nucléoles. Les granulations disparaissent quand la cellule sécrète et possèdent tous les caractères des formations zymogènes.

\section{La paroi parasitée}

C'est aux femelles de Tetrameres qu'est imputable la presque totalité des lésions observées; les mâles n'y contribuent que pour une très faible part. 
A l'œil nu, la présence des parasites se manifeste extérieurement, avant même l'ouverture de l'organe, par de légères bosselures de la paroi ; ces saillies peu accusées sont rendues plus visibles par leur teinte lie de vin, qui tranche sur celle des tissus environnants. On se rend bien compte de cette disposition en examinant la fig. 1 où les deux femelles de Tetrameres déterminent un léger soulèvement des couches musculaires.

En sectionnant ces nodosités, on observe des masses ovoïdes qui s'énucléent facilement d'un seul coup et laissent une cavité correspondant à leurs dimensions. L'examen direct de la muqueuse du proventricule ne permet pas de trouver les vers mâles qui y sont disséminés ; ils sont par contre faciles à découvrir au binoculaire.

En examinant un kyste à la période d'état, contenant une femelle bien développée, on .observe une enveloppe collagène, colorée en rouge intense par la picro-fuchsine ; tapissant intérieurement cette paroi, une mince couche de cellules granuleuses à gros noyaux, enchevêtrées et en désordre : ce sont les vestiges des cellules séreuses à ferments; il ne reste plus trace des cellules muqueuses ; la leucocytose est en général très faible.

La cavité primitive du lobule glandulaire s'est accrue démesurément en suivant la croissance de la femelle de Tetrameres; mais le corp̣s du ver n'est jamais en cóntact avec l'assise résiduaire des cellules granuleuses et un espace vide large de 60 à $300 \mu$ persiste toujours.

Dans la plupart des cas, j'ai constaté la présence d'un mâle logé dans cet espace vide; c'est là un fait nouveau, digne d'être mis en évidence et qui va à l'encontre de l'opinion admise par Raymond, Cram et Travassos : il existe simultanément des mâles libres à la surface de la muqueuse et d'autres profondément enfoncés dans des kystes. On peut constater ce fait sur la fig. 1 qui montre deux sections de ver mâle enkysté. La fig. 2 représente à un plus fort grossissement la même partie de la préparation.

Je n'ai pu observer les stades du début de l'infestation ; il serait intéressant de suivre la pénétration du nématode dans le cul-desac glandulaire et les premières réactions qu'il provoque.

Chez un kyste en voie de développement, les lésions peuvent être schématisées de la façon suivante :

$1^{\circ}$ Dilatation et agrandissement de la capsule conjonctive du lobule ; cette dilatation est particulièrement accusée dans la région où les glandes sont normalement plus petites (pl. XV, fig. 1).

$2^{\circ}$ Tassement et compression mécanique des tubes glandulaires ; cette modification est très visible dans les kystes qui contiennent 
un parasite à demi développé : les travées de cellules granuleuses à ferments cessent d'être orientées radialement; elles se montrent de plus en plus obliques et la lumière des tubes diminue.

$3^{\circ}$ Cytolyse et disparition, d'abord des cellules muqueuses, puis d'une grande partie des cellules séreuses. Ces phénomènes paraissent constituer une véritable digestion du tissu glandulaire par les enzymes de Tetrameres. Dans les cas très avancés, il persiste toujours quelques cellules granuleuses contre la paroi collagène : je n'ai pas vu de lobule intégralement vidé, mais au point de vue physiologique on peut le considérer comme absolument impropre à tout fonctionnement.

$4^{\circ}$ Les couches musculaires du proventricule sont repoussées en saillie légèrement arrondie autour du kyste.

$5^{\circ}$ A côté de ces lésions, faciles à observer dans les lobules envahis, il en est d'autres qui affectent les formations voisines et qui sont la conséquence des premières : les acini intermédiaires sont comprimés et resserrés; leur forme se modifie; elle tend à devenir allongée ; leur paroi repoussée en dedans devient concạve ; mais il ne semble pas que les toxines du ver puissent traverser le sac collagène et agir à distance sur les glandes environnantes. Je n’ai jamais-observé de lésion de nécrose.

Eloïse B. Cram indique (1927) que les femelles de Tetrameres sont toujours orientées dans les kystes avec leur extrémité orale dans le fond et leur région caudale du côté du conduit excréteur du lobe. Cette disposition faciliterait l'émission des œufs. J'ai constaté, comme on peut s'en convaincre par l'examen de la fig. 3, qu'elle est loin d'être générale et que bien souvent l'apex du ver est dirigé vers la cavité du proventricule. Il semble plutôt que les parasites, quand ils sont très nombreux, s'orientent alternativement dans un sens et dans l'autre, pour pouvoir se développer sans trop se gêner.

Les Tetrameres sont fréquents en France chez les oies et les canards (Raymond, 1919); ils le sont beaucoup moins chez les pigeons. Cette étude, en précisant la nature des lésions qu'ils provoquent, fera mieux connaître la gravité de cette helminthiase.

\section{Bibliographie}

CAZIN (M.). - Recherches anatomiques, histologiques et embryologiques sur l'appareil digestif des oiseaux. Ann. Sc. Nat. Zool., $7^{\text {e }}$ série IV, 1888, p. 177-323. 
Cram (Eloïse B.). - Bird parasites of the Nematode suborders Strongylata. Ascaridata and Spirurata. United Statues National Museum, Bull. 140, 1927 , p. 334 (1).

Cattaneo (G.). - Istologia e sviluppo dell apparato gastrico degli uccelli. Atti della soc. Ital. di scienze naturali, XXVII, 1885, p. 90-175.

Masse (C.), - Beiträge zur Histologie des Vogelmagens. Zeitschr. f. rat. Mediz., XXVIII, Hf. 1, 1866.

Leydig (F.). 一 Kleinere Mitteilüngen zur tierischen Geweblehre. Müllers Arch. für Anat., 1854, p. 296-348.

Nüssbaum (Moritz). - Ueber den Bau und die Tätigkeit der Drüsen. Arch. $f$. mikrosk. Anatomie, XIII, 1877, p. 721.

Postma (G.). - Bijdrage tot de kennis van den bouw van het darmkanaal der vogels. Leiden, 1887.

Raymond. - Parasites du tube digestif des oiseaux domestiques. L'hygiène de la viande et du lait, VII, 1919, p. 513.

Travassos (L.). - Contribuiçôes para o conhecimento da fauna helmintolojica brazileira. 3. Sobre as especies brazileiras do genero Tetrameres Creplin 1846. Mem. Inst. Oswaldo Cruz, VII, 1914, p. 146-172, pl. XXIIIXXVI, fig. 1-14 et XI, 1919, p. 71-79, pl. XXV-XXVIII, fig. 1-14.

Wharton Lawhence. - Notes on Nematode parasite of Philippine birds. Tetrameres fissispina (Diesing 1860), in Philippine chickens. Philippine

1. Sc. Manilian, XIII, Sec. D. Gen. Biol. Ethnol. and Anthropol., 1918, p. 219-221.

Laboratoire de Zoologie générale de la Facullé des sciences de Marseille.

(1) E. B. Cram donne une bonne bibliographie jusqu’à 1927.

\section{Explication de la Planche XV}

Fic. 1. - Coupe montrant deux lobules parasités; le plus gros renferme un mâle. A droite, acini normaux. Hématoxyline ferrique. Jaune de métanile. Picrofuchsine. $\times 16$.

Fıg. 2. - Préparation montrant un mâle logé dans le kyste. A droite paroi d'une femelle dont le corps est bourré d'œufs. Hématoxyline ferrique. Jaune de métanile. Picrofuchsine. $\times 290$.

FIG. 3. - Proventricule renfermant de nombreux parasites. On remarquera l'orientation alternée des vers et la compression des acini intermédiaires. Hématoxyline ferrique. Eosine. Orange. $\times 12,5$. 

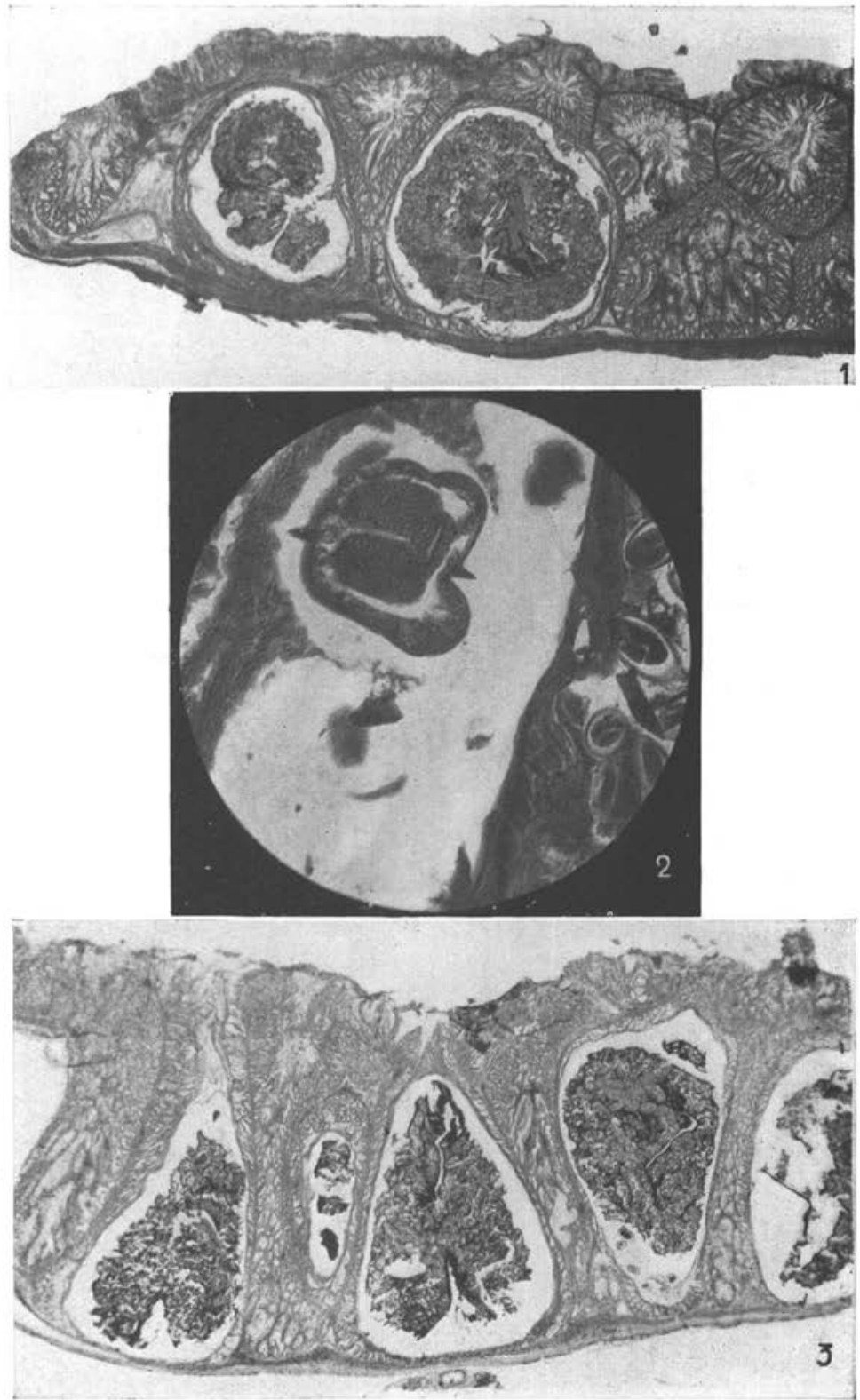